\title{
Atrioventricular optimization in cardiac resynchronization therapy with quadripolar leads
}

Citation for published version (APA):

van Everdingen, W. M., Zweerink, A., Salden, O. A. E., Cramer, M. J., Doevendans, P. A., van Rossum, A. C., Prinzen, F. W., Vernooy, K., Allaart, C. P., \& Meine, M. (2019). Atrioventricular optimization in cardiac resynchronization therapy with quadripolar leads: should we optimize every pacing configuration including multi-point pacing? EP Europace, 21(1), E11-E19. https://doi.org/10.1093/europace/euy138

Document status and date:

Published: 01/01/2019

DOI:

10.1093/europace/euy138

Document Version:

Publisher's PDF, also known as Version of record

Document license:

Taverne

Please check the document version of this publication:

- A submitted manuscript is the version of the article upon submission and before peer-review. There can be important differences between the submitted version and the official published version of record.

People interested in the research are advised to contact the author for the final version of the publication, or visit the DOI to the publisher's website.

- The final author version and the galley proof are versions of the publication after peer review.

- The final published version features the final layout of the paper including the volume, issue and page numbers.

Link to publication

\footnotetext{
General rights rights.

- You may freely distribute the URL identifying the publication in the public portal. please follow below link for the End User Agreement:

www.umlib.nl/taverne-license

Take down policy

If you believe that this document breaches copyright please contact us at:

repository@maastrichtuniversity.nl

providing details and we will investigate your claim.
}

Copyright and moral rights for the publications made accessible in the public portal are retained by the authors and/or other copyright owners and it is a condition of accessing publications that users recognise and abide by the legal requirements associated with these

- Users may download and print one copy of any publication from the public portal for the purpose of private study or research.

- You may not further distribute the material or use it for any profit-making activity or commercial gain

If the publication is distributed under the terms of Article $25 \mathrm{fa}$ of the Dutch Copyright Act, indicated by the "Taverne" license above, 


\title{
Atrioventricular optimization in cardiac resynchronization therapy with quadripolar leads: should we optimize every pacing configuration including multi-point pacing?
}

\author{
Wouter M. van Everdingen ${ }^{1}$, Alwin Zweerink ${ }^{2}$, Odette A. E. Salden ${ }^{1}$, \\ Maarten J. Cramer ${ }^{1}$, Pieter A. Doevendans ${ }^{1}$, Albert C. van Rossum ${ }^{2}$, \\ Frits W. Prinzen ${ }^{3}$, Kevin Vernooy ${ }^{4}$, Cornelis P. Allaart ${ }^{2}$, and Mathias Meine ${ }^{1}$
}

\begin{abstract}
${ }^{1}$ Department of Cardiology, University Medical Center Utrecht, Heidelberglaan 100, 3584 CX Utrecht, The Netherlands; ${ }^{2}$ Department of Cardiology, and Institute for Cardiovascular Research (ICaR-VU), VU University Medical Center, De Boelelaan 1117, 1081 HV Amsterdam, The Netherlands; ${ }^{3}$ Department of Physiology, CARIM, Maastricht University, P. Debyelaan 25, 6229 HX Maastricht, The Netherlands; and ${ }^{4}$ Department of Cardiology, Maastricht University Medical Center, Universiteitssingel 50, 6229 ER Maastricht, The Netherlands
\end{abstract}

Received 11 December 2017; editorial decision 22 May 2018; accepted 14 July 2018; online publish-ahead-of-print 24 July 2018

Aims

This study aims to define an atrioventricular (AV) delay optimization method for cardiac resynchronization therapy (CRT) with a quadripolar left ventricular (LV) lead based on intrinsic conduction intervals.

Methods

and results

Heart failure patients with a left bundle branch block underwent CRT implantation with a quadripolar LV lead. Invasive LV pressure-volume loops were recorded during four biventricular and three multi-point pacing (MPP) settings, using four patient-specific paced AV delays. Haemodynamic response was defined as change in stroke work $(\Delta \% \mathrm{SW})$ compared to intrinsic rhythm and was related to the following conduction intervals: right atrial pacing to right ventricular sensing interval (RAp-RVs), Q to LV sensing interval normalized to QRS duration (QLV/ QRSd), PR-interval, and P-wave duration. In 44 patients, the largest $\Delta \% S W(104 \pm 76 \%)$ occurred at a paced AV delay of $128 \pm 32 \mathrm{~ms}$, at $47 \pm 9 \%$ of RAp-RVs. Optimal AV delay of biventricular pacing $(126 \pm 26 \mathrm{~ms})$ did not differ from MPP (126 $\pm 21 \mathrm{~ms}, P=0.29)$. Intra-class correlation coefficient between optimal AV delays of different pacing configurations was $0.64(0.45-0.78, P<0.001)$. Although not statistically significant, $\Delta \% S W$ at $50 \%$ of RAp-RVs $(98 \pm 74 \%)$ was closer to the maximal achievable $\Delta \% \mathrm{SW}$ increase than a fixed interval of $120 \mathrm{~ms}(96 \pm 73 \%$, $P=0.60)$. RAp-RVs, QLV/QRSd, PR interval, and P-wave duration were associated with the optimal AV delay in univariate analysis, but only RAp-RVs remained significantly associated in multivariate analysis $(R=0.69)$.

Conclusion The AV delay that provides highest haemodynamic response is similar for various LV pacing configurations and for MPP. An AV delay $\sim 50 \%$ of RAp-RVs creates an acute haemodynamic response close to the maximal patientspecific response.

Keywords Cardiac resynchronization therapy - Atrioventricular delay - Pressure-volume loops • Optimization

- Multi-point pacing • Quadripolar lead

\section{Introduction}

Cardiac resynchronization therapy (CRT) is an effective treatment for patients with advanced systolic heart failure and left ventricular
(LV) electrical conduction delay. ${ }^{1}$ Cardiac resynchronization therapy aims to improve LV function with biventricular pacing, leading to electromechanical resynchronization. ${ }^{2}$ Cardiac resynchronization therapy may thereby induce reverse remodelling and may lead to

* Corresponding author. Tel: +31-887550397; fax: +31-887555479. E-mail address: wmvaneverdingen@gmail.com

Published on behalf of the European Society of Cardiology. All rights reserved. @ The Author(s) 2018. For permissions, please email: journals.permissions@oup.com. 


\section{What's new?}

- Atrioventricular (AV) delay optimization is determined based on invasive pressure-volume loops in patients with cardiac resynchronization therapy (CRT).

- The AV delay optimization increases haemodynamic response compared to fixed delays in most patients eligible for CRT with a quadripolar left ventricular (LV) lead.

- The AV delay with highest $\mathrm{LV} \mathrm{dP} / \mathrm{d} t_{\max }$ is significantly longer compared to that providing highest increase in stroke work.

- An AV delay $50 \%$ of the right atrial pacing to right ventricular sensing interval provides an acute haemodynamic response that is close to the maximal patient-specific response.

- The paced AV delay with optimal haemodynamic response is similar for multiple LV pacing configurations and multi-point pacing.

improvements in functional status, exercise tolerance, and subsequently in morbidity and mortality. ${ }^{1}$ These effects are however not seen in all CRT patients, as a considerable amount of patients do not respond significantly. ${ }^{3}$ Non-response to CRT is partly attributed to suboptimal device programming, ${ }^{4}$ which can be optimized using the atrioventricular (AV) delay. The AV delay influences several intracardiac mechanisms that directly impact LV filling, among which atrioventricular interaction is best known. ${ }^{5}$ The $A V$ delay also affects intra- and interventricular interaction ${ }^{5,6}$ and optimization may lead to fusion of intrinsic conduction with either LV and/or right ventricular (RV) pacing.' Optimization of the AV delay can increase acute haemodynamic performance. ${ }^{8}$ Numerous AV delay optimization methods have been proposed, with variable results. ${ }^{9}$ While algorithms using invasive optimization methods may be more reliable, noninvasive methods are more feasible for implementation in clinical practice. 8 A relatively easy, fast, and non-invasive method is the use of intracardiac electrograms (IEGM) to define the AV delay based on measured conduction intervals. ${ }^{10}$ Although IEGM-based algorithms to optimize the AV delay are already included in current devices, most algorithms lack proper physiological support and are at best non-inferior to echocardiographic optimization methods. Moreover, it is unknown whether different LV pacing configurations of a quadripolar $L V$ lead require different $A V$ delays to achieve the maximum potential of CRT.

This study aims to define an AV delay optimization method for CRT with a quadripolar LV lead based on intrinsic conduction intervals. Patient-specific optimal AV delays are determined using pressure-volume (PV) loop analysis, obtained invasively and directly after CRT implantation. The optimal AV delay obtained during multiple pacing configurations of a quadripolar LV lead is compared.

\section{Methods}

This study is part of the OPTICARE-QLV trial, a multicentre observational study performed in three university medical centres in the Netherlands (University Medical Center Utrecht; VU University Medical Center, Amsterdam; and Maastricht University Medical Center, Maastricht), designed to investigate the benefits of quadripolar LV leads in
CRT by invasive PV loop analysis. ${ }^{11}$ In total, 51 consecutive patients with moderate to severe heart failure New York Heart Association (NYHA Class II or III), LV ejection fraction $\leq 35 \%$, sinus rhythm, optimal medical therapy, and a left bundle branch block (LBBB) according to the Strauss criteria were included. ${ }^{12}$ Exclusion criteria were severe aortic valve stenosis, aortic valve replacement, or the presence of LV thrombus. All patients gave written informed consent. The study was performed according to the Declaration of Helsinki and in agreement with the local medical ethics committees.

\section{Study protocol}

An ECG was recorded prior to implantation for all patients, of which PR interval, $\mathrm{P}$ wave duration, QRS duration, and QRS morphology were noted. Patients also underwent echocardiography and cardiac magnetic resonance (CMR) imaging prior to device implantation. Cardiac magnetic resonance or echocardiography-derived LV volumes were used to calibrate the conductance catheter-derived baseline volumes. Cardiac resynchronization therapy implantation was performed under local anaesthesia. Right ventricular and right atrial (RA) leads were placed transvenously at conventional positions. The quadripolar LV lead was aimed at a tributary of the coronary sinus overlying the LV free wall at an anterolateral, lateral, or posterolateral site. After electrophysiological measurements, the three leads were connected to a CRT device.

\section{Electrophysiological measurements}

Electrophysiological measurements were performed using an on-site dedicated system. The electrophysiological system was connected to the surface ECG, and the implanted pacemakers lead to obtain simultaneous recordings. Delays of specific pacing modalities were recorded and delays between pacing spikes and local depolarization were measured. For each patient, the RA sensing to RVs interval (RAs-RVs) and RA pacing to RV sensing interval (RAp-RVs) was measured. For each quadripolar lead electrode, the $\mathrm{Q}$ on surface ECG to local LV depolarization (QLV), QLV normalized for intrinsic QRS duration (QLV/QRSd), and RV pacing to LV sensing interval (RVp-LVs), was measured.

\section{Haemodynamic measurements}

Directly after device implantation, a dedicated PV loop conductance catheter (CD Leycom, Zoetermeer, the Netherlands) was inserted via the femoral artery and placed in the LV cavity. Pressure-volume-loops were recorded for CRT with four paced AV delays during four biventricular pacing settings (BIV, i.e. RV and with one of the four electrodes of the quadripolar LV lead) and three multi-point pacing settings (MPP). The paced $\mathrm{AV}$ delay was set to approximate $80 \%, 60 \%, 40 \%$, and $20 \%$ of the RAp-RVs interval. The measurements were non-randomized, and pacing configurations were programmed in a fixed order, to minimize programming errors. Only the order of the biventricular pacing configurations varied between patients, while the order of atrioventricular delays per pacing configuration was fixed. Biventricular pacing configurations always preceded MPP configurations. The protocol was limited to atrial pacing, to stabilize cardiac rhythm. The interventricular (VV) delay was kept constant at $40 \mathrm{~ms}$ LV first. Pressure-volume-loops of pacing configurations were recorded for 60 beats and 5-10 b.p.m. above intrinsic rhythm, after excluding all inappropriate beats (i.e. extra systoles and two subsequent beats). Pressure-volume-loops during intrinsic conduction (i.e. RA pacing) were recorded as baseline measurements before and after each biventricular pacing run for a period of 30 beats. Change in stroke work (SW) of pacing configurations was calculated as a percentage change ( $\Delta \% \mathrm{SW})$ compared to the mean of the two adjoining baseline measurements. This method allows for reliable assessment of the effects of CRT, by correction of potential baseline drift. ${ }^{13}$ For each BIV and MPP setting, a 

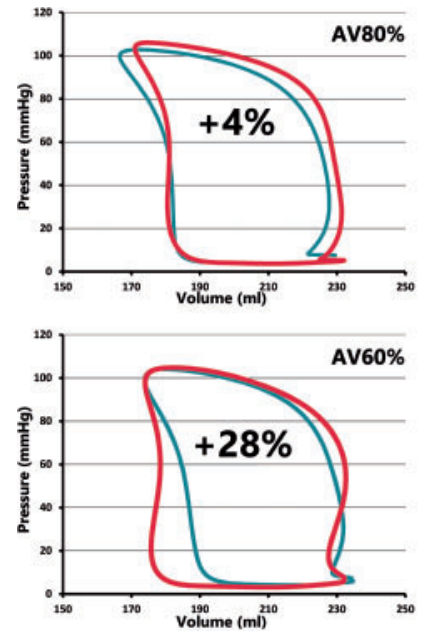
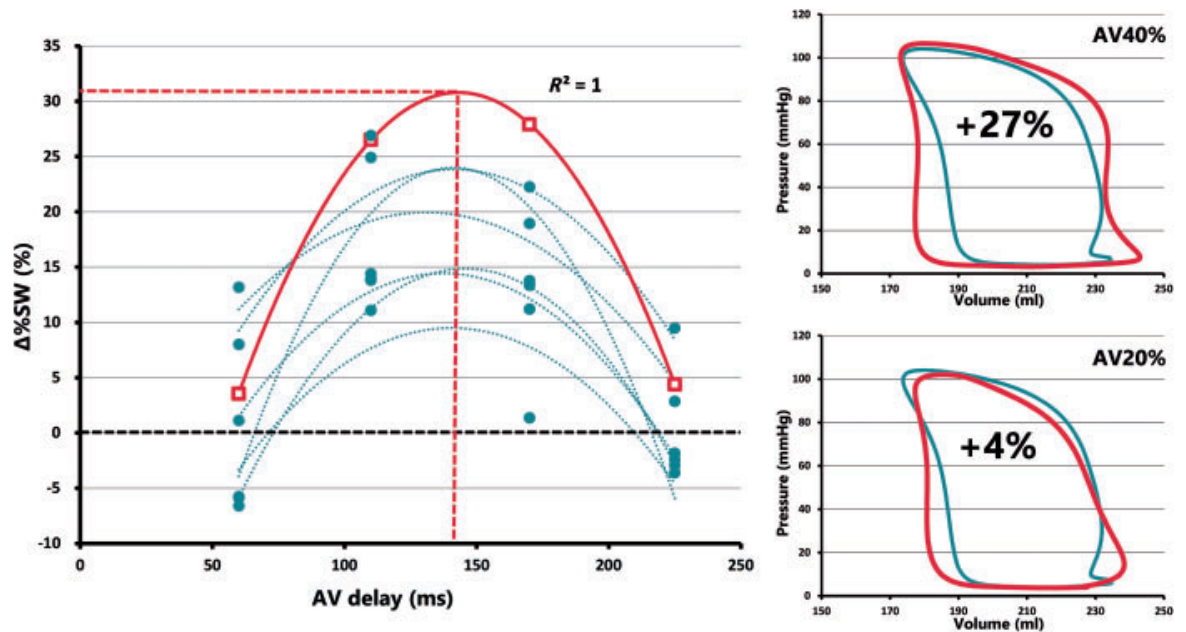

Figure I Method to determine the optimal AV delay. Example of pressure-volume (PV) loops of four AV delays of one pacing configuration using electrode D1. The percentage increase in stroke work $(\Delta \% \mathrm{SW})$ of pacing (red loops) is calculated using the reference loops (blue loops) of right atrial pacing with intrinsic conduction. The results are plotted in a graph, and a 2nd order polynomial line is fitted to the four data points (red parabola). The maximal increase in $\Delta \% \mathrm{SW}$ and corresponding $\mathrm{AV}$ delay are noted. The same is repeated for the remaining six configurations (blue dots and blue dotted curves), resulting in a total of four biventricular pacing configurations and three multi-point pacing configurations. AV, atrioventricular; $\Delta \% \mathrm{SW}$, change in stroke work.

parabolic curve was fitted to the four data points (Figure 1). All fitted curves with a physiological plausible shape (i.e. downward opening with a determinable maximum) and a coefficient of determination $\left(R^{2}\right) \geq 0.7$ were used for further analysis. Of these curves, the maximal increase in $\triangle \% \mathrm{SW}$ and corresponding $\mathrm{AV}$ delay were determined. For each patient, the maximal $\triangle \% \mathrm{SW}$ and corresponding $\mathrm{AV}$ delay $\left(\mathrm{AV}_{\mathrm{OPT}}\right)$ was compared to $\Delta \% \mathrm{SW}$ based on a fixed AV delay of $120 \mathrm{~ms}, 130 \mathrm{~ms}, 160 \mathrm{~ms}$, and $180 \mathrm{~ms}$, determined in the fitted curve. Lastly, the change in $\Delta \% \mathrm{SW}$ based on $50 \%$ of the $R A p-R V s$ delay $\left(A V_{50 \%}\right)$ was calculated using the co-ordinates of the fitted optimization curve (Figure 2).

\section{Statistical analysis}

Statistical analysis was performed using SPSS (SPSS statistics 23.0, IBM, New York, NY, USA). Mean and standard deviation or median and interquartile range are given depended on normality of data. The difference in baseline characteristics between patients in- and excluded in the study was analysed using a Student's $t$-test or Mann-Whitney $U$ test, dependent on normality of data, or a $\chi^{2}$ test in case of categorical variables. Subgroups were compared using similar tests. Optimal AV delays per pacing setting, for SW and $\mathrm{dP} / \mathrm{d} t_{\max }$, for BIV and MPP, and increase in $\Delta \% \mathrm{SW}$ of each $\mathrm{AV}$-optimization strategy were compared using paired $t$-tests. The optimal AV delay per setting was compared with a Pearson correlation coefficient and intra-class correlation coefficient. The univariate relation of predictors for the optimal AV delay were analysed with linear regression analysis. Univariate predictors with a $P$-value $<0.10$ were tested in a multivariate linear regression analysis. A $P$-value below 0.05 was considered significant for all tests.

\section{Ethics}

All procedures performed in studies involving human participants were in accordance with the ethical standards of the institutional and/or national research committee and with the 1964 Helsinki declaration and its later amendments or comparable ethical standards. Informed consent was obtained from all individual participants included in the study.

\section{Results}

Fifty-one patients were included in the main study, of which 44 were used for the present analysis. Reasons for exclusion were: unreliable baseline loops ( $n=3)$, AV optimization curves without a physiological plausible curvature or curves with a low $\left(R^{2}<0.7\right)$ coefficient of determination $(n=4)$. Patients excluded from the analysis had worse diastolic function, with statistically significant higher $E / E^{\prime}$, while left atria tended to be larger (Table 1). In patients included in the final analysis, the paced AV delay with maximal increase in $\triangle \% \mathrm{SW}$ was $128 \pm 32 \mathrm{~ms}$, while the intra-individual variation was $30 \pm 14 \mathrm{~ms}$. Paired $t$-tests showed that there were no statistically significant differences in the optimal AV delay between electrodes (D1: $134 \pm 32 \mathrm{~ms}$, M2: $125 \pm 29 \mathrm{~ms}, \mathrm{M3}: 123 \pm 23 \mathrm{~ms}, \mathrm{P} 4: 123 \pm 24 \mathrm{~ms}$, all $P=$ non-significant). There was also no difference in the optimal $A V$ delay between biventricular pacing $(126 \pm 26 \mathrm{~ms})$ and MPP $(126 \pm 21 \mathrm{~ms}, P=0.29)$. Correlation of the optimal AV delay between the pacing configurations was high (Table 2). Intra-class correlation coefficient for average measures of the optimal AV delay was $0.64(0.45-0.78, P<0.001)$. Examples of AV delay optimization for three patients are depicted in Figure 2. The optimal AV delay lead to an increase in $\Delta \% \mathrm{SW}$ of $104 \pm 76 \%$. The AV delay with maximal increase in $\Delta \% \mathrm{dP} / \mathrm{d} t_{\max }$ was longer compared to the optimal AV delay for $\Delta \% \mathrm{SW}(160 \pm 33$ vs. $128 \pm 32 \mathrm{~ms} P<0.001)$. Atrioventricular optimization led to a mean increase in $\Delta \% \mathrm{dP} / \mathrm{d} t_{\max }$ of $16 \pm 11 \%$ as compared to intrinsic conduction.

\section{Conduction intervals and optimal atrioventricular timing}

A longer optimal paced AV delay was observed in patients with prolonged PR-interval (>200 ms) compared to patients with a normal 

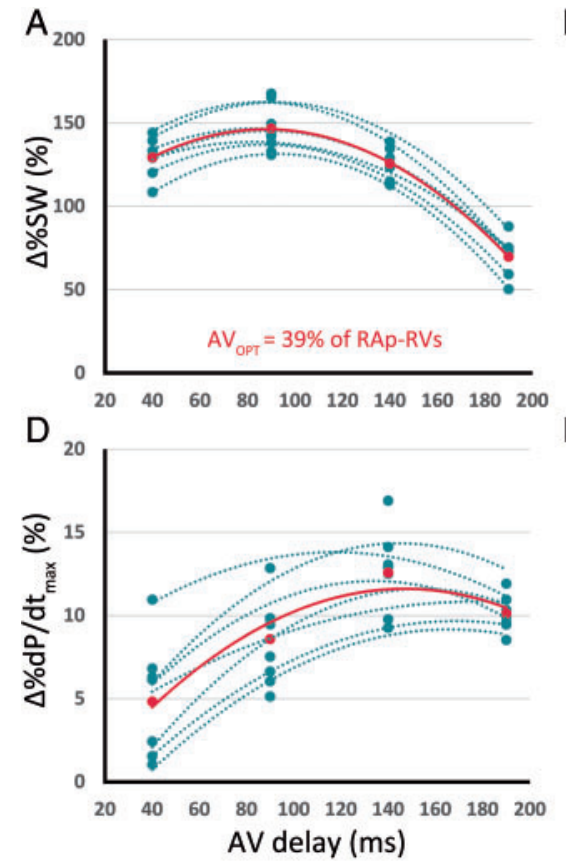

$\mathrm{B}_{80}$

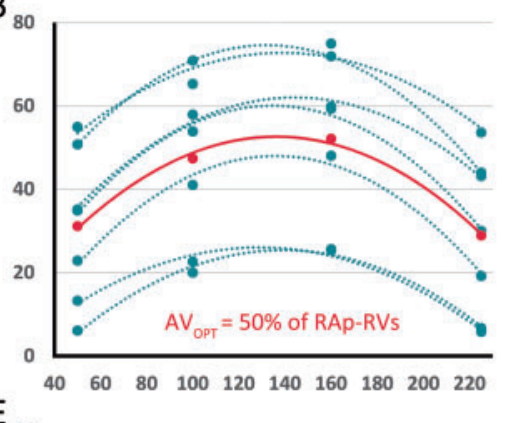

$\mathrm{E}_{20}$

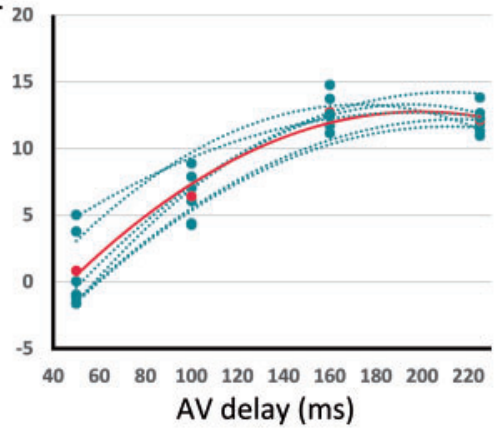

$\mathrm{C}_{250}$

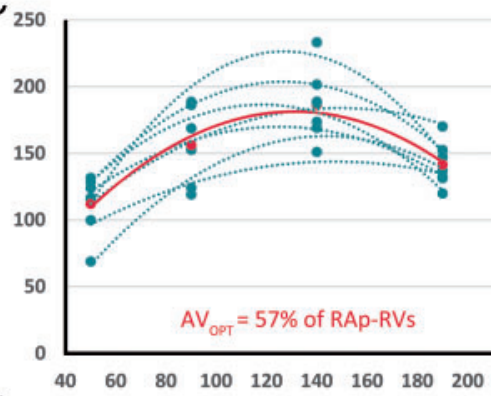

$\mathrm{F}$

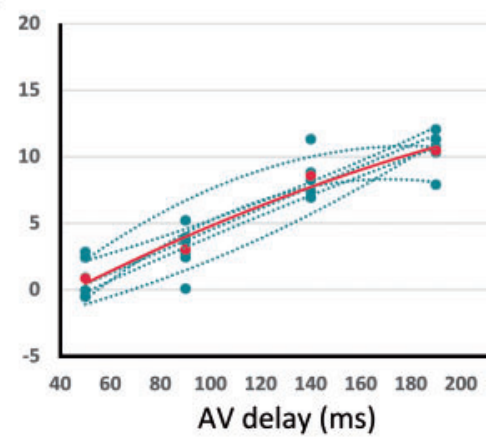

Figure 2 Example of $A V$ delay optimization. Change in stroke work $(\Delta \% \mathrm{SW})$ in the upper panels $(A-C)$ and $\Delta \% \mathrm{dP} / \mathrm{d} t_{\max }$ in the lower panels for three cases $(D-F)$. For each case, up to seven pacing configurations (single- and multi-point) were tested using four $A V$ delays, which are represented by blue dots. The average of all measurements is indicated with black dots. A 2nd order polynomial curve was fitted for each set: pacing configurations (blue dotted curves), average (black dotted curve), and maximum (red solid curve). AV, atrioventricular; $\Delta \% \mathrm{SW}$, change in stroke work.

PR-interval ( $154 \pm 32 \mathrm{~ms}$ vs. $118 \pm 27 \mathrm{~ms}, P=0.001$, Figure 3$)$. Male patients tended to have longer AV delays compared to females (135 \pm 32 vs. $117 \pm 31 \mathrm{~ms}, P=0.08)$. Patients with a NYHA functional Class II also tended to have longer optimal AV delays compared to those NYHA III patients ( $135 \pm 31 \mathrm{~ms}$ vs. $115 \pm 33 \mathrm{~ms}, P=0.05)$. The correlation of ECG derived parameters (i.e. P-wave duration and PRinterval) with the optimal AV delay were all statistically significant (Figure 4). The same accounted for the intracardiac electrogram derived parameters (i.e. RAp-RVs and RAs-RVs). Univariate linear regression showed that several parameters of conduction delay (RAp-RVs, RAs-RVs, P-wave duration, PR interval, and QLV/QRSd) were significantly related to the optimal AV delay (Table 3). The strongest relation was seen between $R A p-R V$ s and $A V_{\text {OPT. }}$. The optimal AV delay was $47 \pm 9 \%$ of the RAp-RVs delay and $4 \pm 29$ ms longer than P-wave duration. The optimal AV delay can be more precisely calculated using the equation: $A V_{\text {OPT }}=1.15 \times R A p-R V_{s}-186 \mathrm{~ms}$. The multivariate linear regression analysis showed that only $R A p-R V s$ remained associated with the optimal AV delay $(R=0.69, P<0.001)$.

\section{Haemodynamic response}

Compared to the other strategies, the change in $\Delta \% \mathrm{SW}$ with $\mathrm{AV}_{50 \%}$ ( $98 \pm 74 \%$ ) was closest to the maximal benefit achievable. The patient-specific effect $A V_{50 \%}$ of showed heterogeneous results, with a considerable amount of patients with an over- or underestimation of the optimal AV delay (Figure 5). Nevertheless, only a four patients with normal PR conduction (PR interval: $156 \mathrm{~ms}$ ) required much shorter AV delays and showed a difference in $\Delta \% \mathrm{SW}$ of $>10 \%$ between $A V_{50 \%}$ and $A V_{\text {OPT }}$. However, the benefit of $A V_{50 \%}$ was small and not significantly different compared to fixed delays of $120 \mathrm{~ms}$ $(96 \pm 73 \%, P=0.29)$ or $130 \mathrm{~ms}(95 \pm 72 \%, P=0.08)$. Nevertheless, pacing at longer $\mathrm{AV}$ delays (i.e. $160 \mathrm{~ms}, 180 \mathrm{~ms}$, and at $70 \%$ of RAp-RVs) showed significantly lower $\Delta \% S W$ values $(85 \pm 66 \%$, $73 \pm 61 \%, 73 \pm 63 \%$, respectively, $P<0.005$ compared to $A V_{50 \%}$, $A V_{120 \mathrm{~ms}}$, and $\left.A V_{130 \mathrm{~ms}}\right)$.

\section{Discussion}

Conduction intervals derived from the intracardiac electrogram can be used to estimate the AV delay with optimal haemodynamic response in patients with CRT. The mean optimal AV delay was dependent on intrinsic conduction delays and corresponded with $\sim 50 \%$ of the RAp-RVs delay. Therefore, an AV delay based on $50 \%$ of the $R A p-R V s$ interval can be used to optimize the AV delay, to achieve an almost optimal, patient specific, effect in haemodynamic response. The AV delay with optimal haemodynamic benefit is similar for all pacing configurations of a quadripolar LV lead, advocating optimization of a single setting before comparison of all possible pacing sites and MPP.

\section{Atrioventricular delay optimization: patient specific or fixed?}

To our knowledge, this is the first study on AV delay optimization in CRT with quadripolar LV leads using PV-loop analysis. Our study showed that the optimal paced AV delay is $\sim 130$ ms determined by 
Table I Baseline characteristics of study population and excluded subjects

\begin{tabular}{|c|c|c|c|}
\hline & Study population $(n=44)$ & Excluded subjects $(n=7)$ & P-value \\
\hline Age (years) & $66 \pm 10$ & $69 \pm 5$ & 0.320 \\
\hline Sex $(\%$ male $)$ & $28(63 \%)$ & $4(57 \%)$ & 0.741 \\
\hline \multicolumn{4}{|l|}{ NYHA class, $n(\%)$} \\
\hline$\|$ & $29(66 \%)$ & $5(71 \%)$ & \multirow[t]{2}{*}{0.774} \\
\hline III & $15(34 \%)$ & $2(29 \%)$ & \\
\hline \multicolumn{4}{|l|}{ Type of CMP, $n(\%)$} \\
\hline DCM & $28(63 \%)$ & $4(57 \%)$ & \multirow[t]{2}{*}{0.741} \\
\hline ICM & $16(36 \%)$ & $3(43 \%)$ & \\
\hline P-wave duration (ms) & $123.5 \pm 12.7$ & $122.0 \pm 18.8$ & 0.776 \\
\hline PR interval (ms) & $184.0 \pm 31.5$ & $179.1 \pm 25.4$ & 0.694 \\
\hline QRS duration (ms) & $175.1 \pm 13.8$ & $178.5 \pm 9.9$ & 0.535 \\
\hline QLV/QRSd (\%) & $83.5 \pm 9.5$ & $87.9 \pm 4.3$ & 0.246 \\
\hline LV EDV (mL) & $198(169-241)$ & $240(184-304)$ & 0.268 \\
\hline LV ESV (mL) & $148(106-172)$ & $180(136-252)$ & 0.234 \\
\hline LV EF (\%) & $28.9 \pm 8.4$ & $23.4 \pm 6.5$ & 0.105 \\
\hline E/A ratio & $0.65(0.49-0.99)$ & $1.30(0.72-1.50)$ & 0.118 \\
\hline$E / E^{\prime}$ & $12.2(10.1-16.0)$ & $18.4(13.7-27.1)$ & 0.042 \\
\hline LA size $\left(\mathrm{mL} / \mathrm{m}^{2}\right)$ & $35.7(28.2-41.2)$ & $40.8(35.1-60.2)$ & 0.052 \\
\hline${ }^{10} \log \mathrm{BNP}(\mathrm{pmol} / \mathrm{L})$ & $1.87 \pm 0.56$ & $2.15 \pm 0.47$ & 0.219 \\
\hline Creatinine $(\mu \mathrm{mol} / \mathrm{L})$ & $90.4 \pm 23.5$ & $100.6 \pm 41.2$ & 0.549 \\
\hline RAp-RVs (ms) & $274.3 \pm 49.1$ & $273.6 \pm 23.4$ & 0.970 \\
\hline RAs-RVs (ms) & $201.9 \pm 31.4$ & $202.1 \pm 47.4$ & 0.985 \\
\hline $\mathrm{QLV}_{\max }(\mathrm{ms})$ & $146.2 \pm 19.7$ & $156.9 \pm 11.8$ & 0.172 \\
\hline QLV/QRSd (\%) & $83.5 \pm 9.5$ & $87.9 \pm 4.3$ & 0.246 \\
\hline$R V_{p}-L V_{s_{\max }}(m s)$ & $157.6 \pm 24.9$ & $173.9 \pm 23.0$ & 0.112 \\
\hline Optimal AV delay for SW (ms) & $128.0 \pm 32.2$ & $126.8 \pm 59.6^{\mathrm{a}}$ & 0.940 \\
\hline Optimal $\mathrm{AV}$ delay for $\mathrm{dP} / \mathrm{d} t_{\max }(\mathrm{ms})$ & $159.5 \pm 33.0$ & $158.7 \pm 23.1$ & 0.950 \\
\hline
\end{tabular}

LV volumes and ejection fraction are based on echocardiography.

$\mathrm{AV}$, atrioventricular; BNP, brain natriuretic peptide; CMP, cardiomyopathy; DCM, dilated cardiomyopathy; ECG, electrocardiogram; EDV, end-diastolic volume; ESV, endsystolic volume; EF, ejection fraction; ICM, ischaemic cardiomyopathy; LA, left atrial; LV, left ventricular; NYHA, New York Heart Association; QLV max, maximal delay between Q on surface ECG and LV depolarization; QLV/QRSd, ratio between QLV and intrinsic QRS duration; RAp-RVs, right atrial pacing to right ventricular sensing interval; RAs-RVs, right atrial sensing to right ventricular sensing interval; $R V_{p}-L_{s_{\text {max }}}$, maximal conduction interval between right ventricular pacing to left ventricular sensing; SW, stroke work. a Some patients were excluded because of unreliable baseline loops and still showed $\mathrm{AV}$ optimization curves with a physiological shape and $R^{2}>0.7$.

Bold P-values are below 0.05 and therefore statistically significant.

Table 2 Correlation of optimal atrioventricular delays between pacing configurations

\begin{tabular}{|c|c|c|c|c|c|c|c|}
\hline & BIV1 & BIV2 & BIV3 & BIV4 & MPP1 & MPP2 & MPP3 \\
\hline BIV1 & & $0.690 *$ & $0.872 *$ & $0.763^{*}$ & $0.833^{*}$ & $0.895^{*}$ & $0.668 *$ \\
\hline BIV2 & $0.690 *$ & & $0.878 *$ & $0.806^{*}$ & $0.885^{*}$ & $0.836^{*}$ & $0.732 *$ \\
\hline BIV3 & $0.872 *$ & $0.878 *$ & & $0.785^{*}$ & $0.951 *$ & $0.925^{*}$ & $0.796 *$ \\
\hline BIV4 & $0.763 *$ & $0.806 *$ & $0.785^{*}$ & & $0.916 *$ & $0.871 *$ & $0.791 *$ \\
\hline MPP1 & $0.833 *$ & $0.885^{*}$ & $0.951 *$ & $0.916^{*}$ & & $0.940^{*}$ & $0.850 *$ \\
\hline MPP2 & $0.895^{*}$ & $0.836^{*}$ & $0.925^{*}$ & $0.871 *$ & $0.940 *$ & & $0.498^{\dagger}$ \\
\hline MPP3 & $0.668 *$ & $0.732 *$ & $0.796 *$ & $0.791 *$ & $0.850 *$ & $0.498^{\dagger}$ & \\
\hline
\end{tabular}

Matrix of Pearson correlation coefficients of the optimal atrioventricular delays for each pacing configuration. BIV1 to -4 are biventricular pacing configurations with one of the quadripolar electrodes. MPP1 to -3 are multi-point pacing configurations.

$* P<0.001$;

${ }^{\dagger} P<0.05$. 


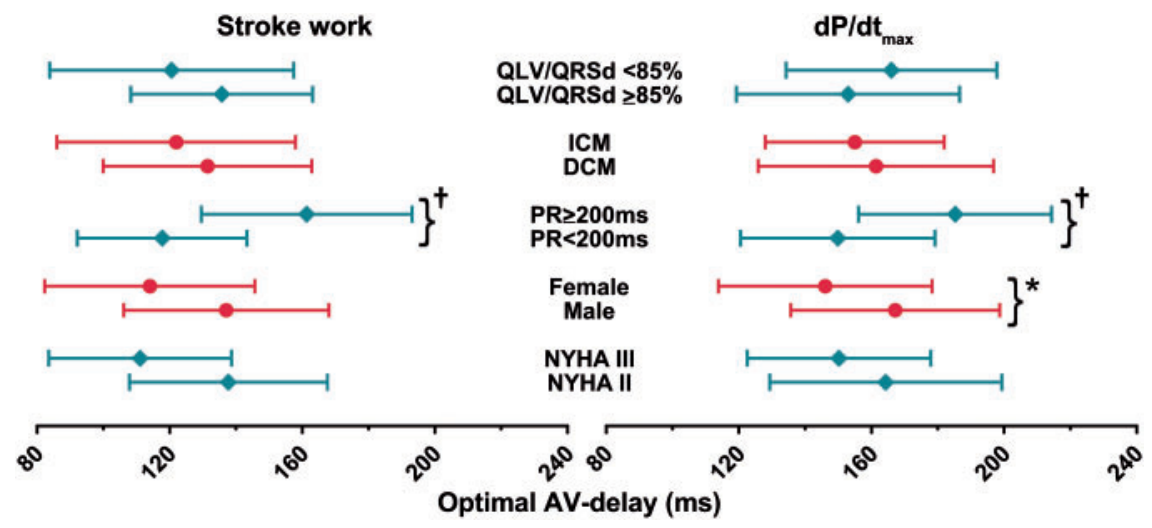

Figure 3 Optimal atrioventricular delay of specific subgroups. Atrioventricular (AV) delay with optimal haemodynamic response measured as change in stroke work and $\mathrm{dP} / \mathrm{d} t_{\max }$. Categories include the QLV/QRSd, type of cardiomyopathy: DCM and ICM. PR interval above or below the cutoff value for 1st degree AV block (i.e. $200 \mathrm{~ms}$ ), gender, and type of NYHA functional class. Statistical significant difference with $* P<0.05$; ${ }^{\dagger} P<0.002$. $\mathrm{AV}$, atrioventricular; DCM, dilated cardiomyopathy; ICM, ischaemic cardiomyopathy; QLV/QRSd, ratio between $\mathrm{Q}$ to left ventricular sensing delay and QRS duration ratio; NYHA, New York Heart Association.

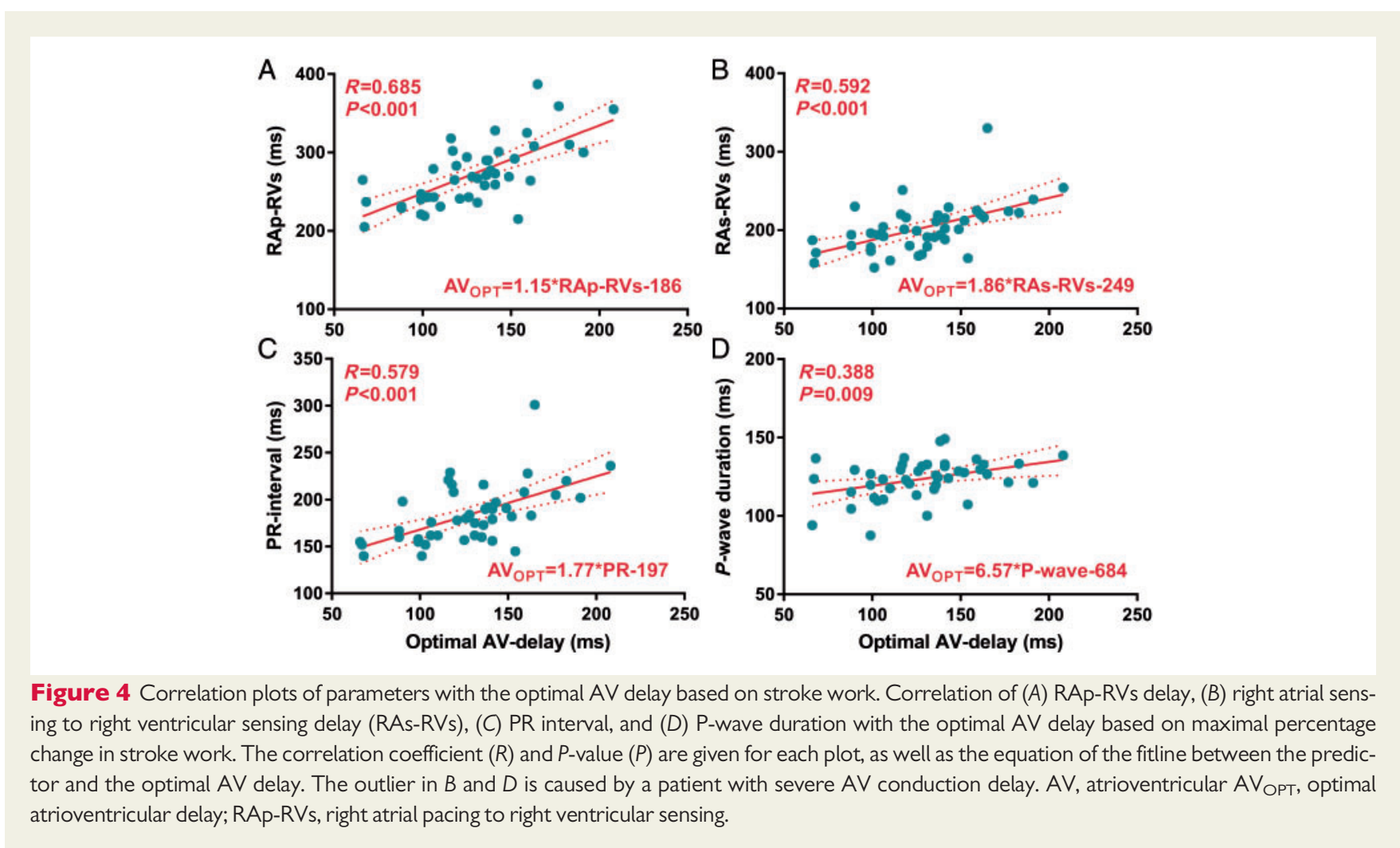

$\Delta \% \mathrm{SW}$ and $\sim 160 \mathrm{~ms}$ by $\mathrm{LV} \mathrm{dP} / \mathrm{d} t_{\max }$, which is shorter than earlier results of our group (i.e. optimal paced $A V$ delay with $L V d P / d t_{\max }$ of $180 \mathrm{~ms}) .^{8}$ The difference between RAs-RVs and RAp-RVs was $\sim 70 \mathrm{~ms}$ in this study and implementation of the $A V_{50 \%}$ algorithm would result in an optimal sensed AV delay $35 \mathrm{~ms}$ shorter than the paced AV delay (i.e. $\sim 100 \mathrm{~ms}$ ). The sensed AV delay would be shorter compared to values found in previous studies (i.e. SMART-
AV: $120 \mathrm{~ms}$ ) and work of our own group (i.e. $130 \mathrm{~ms}) .{ }^{8,9}$ However, those results were based on $L V d P / d t_{\max }$, which only reflects the rate of LV pressure changes in the isovolumetric contraction phase. Left ventricular $\mathrm{dP} / \mathrm{d} t_{\max }$ is highest at fusion of intrinsic conduction and ventricular pacing, which occurs at relative long AV delays, ${ }^{8}$ despite reduced diastolic filling properties. Results on PV-loops incorporate information on pressure and volume changes throughout the entire 
Table 3 Univariate and multivariate relation of predictors for the optimal AV delay

\begin{tabular}{|c|c|c|c|c|c|c|}
\hline \multirow[b]{2}{*}{ Variable } & \multicolumn{3}{|c|}{ Univariate } & \multicolumn{3}{|c|}{ Multivariate } \\
\hline & B (SD) & $R$ & P-value & B (SD) & $R$ & $P$-value \\
\hline RAp-RVs (ms) & $0.55(0.09)$ & 0.69 & $<0.001$ & $0.54(0.09)$ & 0.69 & $<0.001$ \\
\hline RAs-RVs (ms) & $0.60(0.13)$ & 0.59 & $<0.001$ & & & 0.977 \\
\hline P-wave duration (ms) & $0.99(0.36)$ & 0.39 & 0.009 & & & 0.167 \\
\hline PR interval (ms) & $0.59(0.13)$ & 0.58 & $<0.001$ & & & 0.190 \\
\hline QRS duration (ms) & $-0.09(0.36)$ & 0.04 & 0.800 & & & . \\
\hline QLV (ms) & $-0.43(0.24)$ & 0.26 & 0.087 & & & . \\
\hline QLV/QRSd (\%) & $-1.01(0.50)$ & 0.30 & 0.049 & & & 0.343 \\
\hline$R V_{p}-L V_{s}(m s)$ & $-0.05(0.20)$ & 0.04 & 0.797 & & & . \\
\hline
\end{tabular}

$\mathrm{AV}$, atrioventricular; B, Beta coefficient; QLV, Q on surface ECG and LV depolarization; QLV/QRSd, ratio between $\mathrm{Q}$ to left ventricular sensing delay and QRS duration ratio $\mathrm{R}$, correlation coefficient; RAp-RVs, right atrial pacing to right ventricular sensing; RAs-RVs, right atrial sensing to right ventricular sensing; $R V_{p}-L V s$, right ventricular pacing to left ventricular sensing interval; $S D$, standard deviation.

Bold $P$-values are below 0.05 and therefore statistically significant.

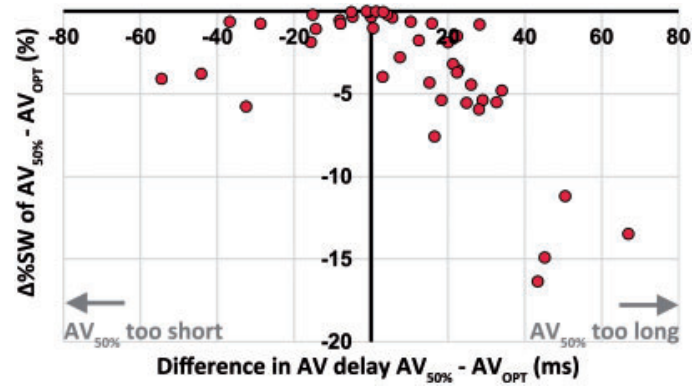

Figure 5 Difference in stroke work and atrioventricular delay of $\mathrm{AV}_{50}$ vs. $\mathrm{AV}$ OPT. The patient-specific effect of an $\mathrm{AV}$ delay based $50 \%$ of the right atrial pacing to right ventricular sensing interval $\left(\mathrm{AV}_{50 \%}\right)$ compared to the $A V_{\text {OPT }}$. Difference between $A V_{50 \%}$ and $A V_{\text {OPT }}$ on $\Delta \% \mathrm{SW}$ and the $\mathrm{AV}$ delay is given. $\mathrm{AV}$, atrioventricular; $\mathrm{AV}$ OPT, optimal atrioventricular delay; $\Delta \% \mathrm{SW}$, change in stroke work.

cardiac cycle, and thus include the systolic and diastolic performance. Shortening of the AV delay during CRT will lead to an increase in preload, as shown by Jones et al., ${ }^{14}$ which is directly visualized in a PV-loop. The effect of increased preload is reflected by the shorter optimal AV delays found with $\Delta \% \mathrm{SW}$ compared to $\mathrm{LV} \mathrm{dP} / \mathrm{d} t_{\max }$. Some patients in our study benefited in terms of $\Delta \% \mathrm{SW}$ with rather short AV delays. These short AV delays will decrease the effect of intrinsic conduction on ventricular activation, which indicates that interventricular interaction due to $L V$ and $R V$ pacing is more important in some patients. These heterogeneous effects, even in a cohort of patients with LBBB, show that there is no 'one size fits all' method for AV optimization. The observation that the optimal AV delay is close to $50 \%$ of RAp-RVs indicates that filling parameters may be important. Since absolute values of the optimal AV delay are longer for patients with prolonged PR intervals, optimized atrioventricular filling seems more important than fusion of intrinsic conduction and LV and $\mathrm{RV}$ pacing in most patients. The RAp-RVs is moreover influenced by the position of the RV lead. Although all leads were positioned towards the RV apex, small differences in RV lead position might have influenced the correlation between RAp-RVs and the optimal AV delay. The mean AV delay for RV pacing in our study (50\% of $R A p-R V s+40 \mathrm{~ms}=177 \mathrm{~ms}$ ) was also shorter than the intrinsic PR interval (184 ms). The association between PR interval and optimal AV delay may be ascribed to conduction delay in the atria, requiring a longer interval between complete atrial activation and ventricular activation. This is supported by the association between $\mathrm{P}$ wave duration and the optimal AV delay. As seen in prior studies, ${ }^{8}$ a trend towards a longer PR interval is seen in men compared to women. Men have larger hearts compared to women, indicating that cardiac size influences optimal AV timing. The effect of CRT in patients with prolonged PR interval is of interest, as a benefit was found in a sub analysis of the COMPANION trial and in a sub-analysis of non-LBBB patients in the MADIT-CRT. ${ }^{15,16}$ In these sub analyses, patients with a prolonged PR interval benefited more from CRT in terms of reduced heart failure hospitalizations and mortality. ${ }^{17}$

\section{Comparison to current device algorithms}

All CRT devices have built in algorithms for AV delay optimization based on intracardiac electrograms. Patients may benefit more from CRT with the $A V_{50 \%}$ method, as current algorithms do not shorten the AV delay far enough. Current methods, such as QuickOpt (St. Jude Medical, St. Paul, Minnesota, USA), rely on a fixed sum of several milliseconds on measured $\mathrm{P}$-wave duration (i.e. $80 \mathrm{~ms}$ if $\mathrm{P}$-wave duration $<100$ and $110 \mathrm{~ms}$ if $\mathrm{P}$-wave duration $>100 \mathrm{~ms}$ ). In our study population, QuickOpt would result in relatively long paced AV delays (i.e. $205 \pm 10 \mathrm{~ms}$ ). Moreover, the increase with $30 \mathrm{~ms}$ based on a $\mathrm{P}$ wave duration above $100 \mathrm{~ms}$ is not physiological. ${ }^{13}$ The Adaptiv-CRT algorithm of Medtronic (Minneapolis, MN, USA) implements LV only pacing and uses an AV delay at $70 \%$ of the RAp-RVs delay or RApRVs -40 ms (i.e. whichever is shorter, and the paced AV delay never exceeds $180 \mathrm{~ms}$ ), for fusion with intrinsic conduction. Adaptiv-CRT has been proven to be effective on clinical outcome and echocardiographic response. ${ }^{10,18}$ The Adaptiv-CRT algorithm is different for patients with a RAp-RVs interval above or below $270 \mathrm{~ms}$ (or $250 \mathrm{~ms}$ in other Medtronic devices). However, the optimal AV delay in the 
24 patients with RAp-RVs $\leq 270 \mathrm{~ms}$ in our study was significantly shorter compared to Adaptiv-CRT (i.e. $111 \mathrm{~ms}$ vs. Adaptiv-CRT: $168 \mathrm{~ms})$. The AV optimization method described by Gold et al. ${ }^{19}$ closely resembles the $A V_{50 \%}$ strategy, as it also uses a fraction of measured pacing intervals between atria and ventricles. Nevertheless, the resulting paced $\mathrm{AV}$ delays of Gold et al. ${ }^{19}$ were longer (i.e. $208 \pm 62 \mathrm{~ms}$ ) than ours. The difference between prior mentioned optimization algorithms and our results suggest that $L V d P / d t_{\max }$ measurements were used for these algorithms.

As most of these algorithms, AV delay optimization with the RAp$R V$ s interval facilitates continuous AV optimization after implantation. Atrioventricular conduction may be influenced by LV reverse remodelling and changes in pharmacological therapy after CRT. Changes in the atrioventricular conduction may influence RAp-RVs and subsequent programmed AV delay for optimal haemodynamic response. Periodic optimization of the AV delay by periodic measurements of the RAp-RVs may therefore be of additional benefit.

\section{Limitations}

There are some limitations to take into account. The AV delay was optimized directly after device implantation and translation of these results to $A V$ delay optimization after evidence of reverse remodelling is unknown. The benefit of patient-specific AV delay optimization on long-term benefit of CRT is controversial. There is no clear benefit of any chosen strategy compared to fixed AV delays. ${ }^{20}$ Nevertheless, increase in $\Delta \% \mathrm{SW}$ is known to predict long-term CRT response, ${ }^{21}$ and every percentage of increase in $\Delta \% \mathrm{SW}$ may potentially improve the patient's prognosis. Measurements were performed at rest, which may influence optimal AV timing. Exercise, and thereby increased sympathetic drive, leads to an increase in haemodynamic function, an increased heart rate and relative shorter diastolic phase compared to the systolic phase. A rate-adaptive approach may therefore be of benefit to haemodynamic response, as intrinsic $A V$ conduction shortens during exercise. Implementation of the proposed method $\left(A V_{50 \%}\right)$ in a rate-adaptive algorithm is of interest for future studies. Atrioventricular delay optimization was performed with a fixed $\mathrm{VV}$ delay of $40 \mathrm{~ms}$ LV first because previous studies showed that LV pre-activation produces the highest haemodynamic response. 8 To avoid programming errors, the pacing protocols were applied in a fixed order and were therefore nonrandomized. As the implemented optimization protocol was time consuming and to stabilize heart rhythm, only optimization of the paced AV delay was performed. However, CRT patients are generally in sinus rhythm, and ventricular pacing after atrial sensing is more common in clinical practice. Although there was no significant benefit of an IEGM-based method compared to fixed AV delays, this study might have been underpowered to find a significant difference between strategies. Lastly, comparison to AV delay optimization strategies based on shortening of the QRS complex or QRS area on vector cardiography are of interest. ${ }^{22}$ However, these optimization strategies went beyond the scope of this article.

\section{Conclusion}

A paced AV delay optimization strategy based on $50 \%$ of the intrinsic $\mathrm{AV}$ conduction interval is closest to the maximal achievable and patient-specific acute haemodynamic effect. As the AV delay for optimal haemodynamic response is similar between pacing configurations of a quadripolar LV lead in a single patient, optimization is only necessary for one pacing configuration.

\section{Funding}

This study was conducted with an unrestricted research grant from St. Jude Medical (St. Paul, MN, USA).

Conflict of interest: M.M. has received research grants from Boston Scientific and St. Jude Medical. K.V. has received speaker fees and research grants from St. Jude Medical. F.W.P. has received research grants from Medtronic Inc., Boston Scientific Corp., St. Jude Medical, LivaNova, Biosense Webster, and EBR Systems and is advisor to Medtronic Inc. All the remaining authors declare that they have no conflict of interests.

\section{References}

1. Cleland JG, Abraham WT, Linde C, Gold MR, Young JB, Claude Daubert J et al. An individual patient meta-analysis of five randomized trials assessing the effects of cardiac resynchronization therapy on morbidity and mortality in patients with symptomatic heart failure. Eur Heart J 2013;34:3547-56.

2. Prinzen FW, Vernooy K, De Boeck BWL, DeBoeck BWL, Delhaas T. Mechanoenergetics of the asynchronous and resynchronized heart. Heart Fail Rev 2011; 16:215-24.

3. Daubert J-C, Saxon L, Adamson PB, Auricchio A, Berger RD, Beshai JF et al. 2012 EHRA/HRS expert consensus statement on cardiac resynchronization therapy in heart failure: implant and follow-up recommendations and management. Europace 2012;14:1236-86.

4. Mullens W, Grimm RA, Verga T, Dresing T, Starling RC, Wilkoff BL et al. Insights from a cardiac resynchronization optimization clinic as part of a heart failure disease management program. J Am Coll Cardiol 2009;53:765-73.

5. Auricchio A, Ding J, Spinelli JC, Kramer AP, Salo RW, Hoersch W et al. Cardiac resynchronization therapy restores optimal atrioventricular mechanical timing in heart failure patients with ventricular conduction delay. J Am Coll Cardiol 2002;39: 1163-9.

6. Sweeney MO, Prinzen FW. Ventricular pump function and pacing: physiological and clinical integration. Circ Arrhythm Electrophysiol 2008;1:127-39.

7. Vernooy K, Verbeek XA, Cornelussen RN, Dijkman B, Crijns HJ, Arts T et al. Calculation of effective $\mathrm{VV}$ interval facilitates optimization of $A V$ delay and $\mathrm{V} V$ interval in cardiac resynchronization therapy. Heart Rhythm 2007;4:75-82.

8. Bogaard MD, Meine M, Tuinenburg AE, Maskara B, Loh P, Doevendans PA. Cardiac resynchronization therapy beyond nominal settings: who needs individual programming of the atrioventricular and interventricular delay? Europace 2012;14:1746-53.

9. Ellenbogen KA, Gold MR, Meyer TE, Fernndez Lozano I, Mittal S, Waggoner AD et al. Primary results from the SmartDelay determined AV optimization: a comparison to other AV delay methods used in cardiac resynchronization therapy (SMART-AV) trial: a randomized trial comparing empirical, echocardiographyguided, and algorithmic atrioventricular delay programming in cardiac resynchronization therapy. Circulation 2010;122:2660-8.

10. Singh JP, Abraham WT, Chung ES, Rogers T, Sambelashvili A, Coles JA Jr et al. Clinical response with adaptive CRT algorithm compared with CRT with echocardiography-optimized atrioventricular delay: a retrospective analysis of multicentre trials. Europace 2013;15:1622-8.

11. van Everdingen WM, Zweerink A, Cramer MJ, Doevendans PA, Nguyen UC, Van Rossum AC et al. Can we use the intrinsic left ventricular delay (QLV) to optimize the pacing configuration for cardiac resynchronization therapy with a quadripolar left ventricular lead? Circ Arrhythm Electrophysiol 2018;11:e005912.

12. Strauss DG, Selvester RH, Wagner GS. Defining left bundle branch block in the era of cardiac resynchronization therapy. Am J Cardiol 2011;107:927-34.

13. Sohaib SM, Whinnett Zl, Ellenbogen KA, Stellbrink C, Quinn TA, Bogaard MD et al. Cardiac resynchronisation therapy optimisation strategies: systematic classification, detailed analysis, minimum standards and a roadmap for development and testing. Int J Cardiol 2013;170:118-31.

14. Jones S, Lumens J, Sohaib SM, Finegold JA, Kanagaratnam P, Tanner $M$ et al. Cardiac resynchronization therapy: mechanisms of action and scope for further improvement in cardiac function. Europace 2017;19:1178-86.

15. Olshansky B, Day JD, Sullivan RM, Yong P, Galle E, Steinberg JS. Does cardiac resynchronization therapy provide unrecognized benefit in patients with prolonged PR intervals? The impact of restoring atrioventricular synchrony: an analysis from the COMPANION Trial. Heart Rhythm 2012;9:34-9. 
16. Kutyifa V, Stockburger M, Daubert JP, Holmqvist F, Olshansky B, Schuger C et al. PR interval identifies clinical response in patients with non-left bundle branch block: a Multicenter Automatic Defibrillator Implantation Trial-Cardiac Resynchronization Therapy substudy. Circ Arrhythm Electrophysiol 2014;7: 645-51.

17. Rickard J, Karim M, Baranowski B, Cantillon D, Spragg D, Tang WHW et al. Effect of PR interval prolongation on long-term outcomes in patients with left bundle branch block vs non-left bundle branch block morphologies undergoing cardiac resynchronization therapy. Heart Rhythm 2017;14:1523-8.

18. Burns KV, Gage RM, Curtin AE, Gorcsan J 3rd, Bank AJ. Left ventricular-only pacing in heart failure patients with normal atrioventricular conduction improves global function and left ventricular regional mechanics compared with biventricular pacing: an adaptive cardiac resynchronization therapy sub-study. Eur J Heart Fail 2017;19:1335-43.
19. Gold MR, Niazi I, Giudici M, Leman RB, Sturdivant JL, Kim MH et al. A prospective comparison of AV delay programming methods for hemodynamic optimization during cardiac resynchronization therapy. J Cardiovasc Electrophysiol 2007;18: 490-6.

20. Auger D, Hoke U, Bax JJ, Boersma E, Delgado V. Effect of atrioventricular and ventriculoventricular delay optimization on clinical and echocardiographic outcomes of patients treated with cardiac resynchronization therapy: a meta-analysis. Am Heart J 2013;166:20-9.

21. de Roest GJ, Allaart CP, Kleijn SA, Delnoy PP, Wu L, Hendriks ML et al. Prediction of long-term outcome of cardiac resynchronization therapy by acute pressure-volume loop measurements. Eur J Heart Fail 2013;15:299-307.

22. van Deursen C], Wecke L, van Everdingen WM, Stahlberg $M$, Janssen $M H$, Braunschweig $\mathrm{F}$ et al. Vectorcardiography for optimization of stimulation intervals in cardiac resynchronization therapy. J Cardiovasc Transl Res 2015;8:128-37. 